\title{
Red clump giant stars as tracers of Galactic structure
}

\author{
M. López-Corredoira ${ }^{1}$, A. Cabrera-Lavers ${ }^{2}$, P. L. Hammersley ${ }^{1}$, \\ F. Garzón ${ }^{1,3}$, T. J. Mahoney ${ }^{1}$ and C. González-Fernández ${ }^{1}$ \\ ${ }^{1}$ Instituto de Astrofísica de Canarias, La Laguna (Tenerife, Spain) \\ ${ }^{2}$ GRANTECAN, La Palma (Spain) \\ ${ }^{3}$ Departamento de Astrofísica, Universidad de La Laguna (Tenerife, Spain)
}

\begin{abstract}
By isolating the red clump giant population in the color-magnitude diagrams and inverting their star counts, we can obtain directly the density distribution of the old stellar population along the line of sight. We have applied this method to several near infrared surveys and obtained information on the disc, bulge and long bar. The disc is well fitted by an exponential distribution in both the galactocentric distance and height, flared and warped in the outer parts, and with a deficit of stars in the inner in-plane regions. The long bar occupies these in-plane regions within $R<3.9 \mathrm{kpc}$, with approximate dimensions of $7.8 \mathrm{kpc} \times 1.2 \mathrm{kpc} \times 0.2 \mathrm{kpc}$ and a position angle of $40-45 \mathrm{deg}$. The bulge is a triaxial structure, possibly boxy, thicker and shorter than the long bar and with position angle of 10-30 deg.
\end{abstract}

Keywords. Galaxy: structure, infrared: stars

The red clump giants (approx. K0-2III stars) constitute a very prominent population in the near infrared color magnitude diagrams (CMDs). Their absolute magnitude is $M_{K} \approx-1.65$, and intrinsic color $J-K \approx 0.75$, so their property as standard candles can be used to derive the parameters of the Galactic structure. A method, explained in detail in López-Corredoira et al. (2002, Sect. 3), allows us to obtain the star density along a line of sight $(l, b)$ from an analysis of near-infrared CMDs, such as $m_{K}$ vs. $(J-K)$. First, the trace produced in the CMD by the red clump giants is identified. Second, we count the number of stars within a fixed width trace (we usually take $0.4 \mathrm{mag}$ ) as a function of the apparent magnitude. Third, since absolute magnitude and intrinsic color are known, we can obtain the extinction and density as a function of distance along the line of sight.

The method was applied to derive the parameters of the thin disc (López-Corredoira et al. 2002, 2004), thin+thick disc (Cabrera-Lavers et al. 2007a), long bar+thick bulge (Cabrera-Lavers et al. 2007b and references therein). The general obtained picture is that the disc is flared in the outer and inner parts, and without cut-off up to $16 \mathrm{kpc}$; and that the central $4 \mathrm{kpc}$ of the Galaxy contains a double triaxial structure: long bar and thick bulge with position angles of 40-45 and 10-30 deg. respectively.

\section{References}

López-Corredoira, M., Cabrera-Lavers, A., Garzón, F., \& Hammersley, P. L. 2002, A 8 A 394, 883

López-Corredoira, M., Cabrera-Lavers, A., Gerhard, O. E., \& Garzón, F. 2004, A $\mho 3 A$ 421, 953

Cabrera-Lavers, A., Bilir, S., Ak, S., Yaz, E., \& López-Corredoira, M. 2007a, A $\& A$ A 464, 565

Cabrera-Lavers, A., Hammersley, P. L., González-Fernández, C., López-Corredoira, M., Garzón, F., \& Mahoney, T. J. 2007b, A\&̈A 465, 825 